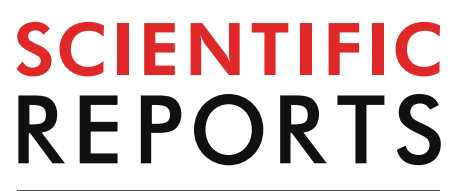

natureresearch

Check for updates

\title{
Micro-rheological properties
} of lung homogenates correlate with infection severity in a mouse model of Pseudomonas aeruginosa lung infection

Xabier Murgia ${ }^{1,6,7}$, Andreas M. Kany ${ }^{2,3,7}$, Christian Herr ${ }^{4}$, Duy-Khiet Ho ${ }^{1}$, Chiara De Rossi ${ }^{1}$ Robert Bals ${ }^{4}$, Claus-Michael Lehr ${ }^{1,5}$, Anna K. H. Hirsch ${ }^{2,3,5}$, RolfW. Hartmann ${ }^{2,3,5}$, Martin Empting ${ }^{2,3,5 \bowtie}$ \& Teresa Röhrig ${ }^{2,3 凶}$

Lung infections caused by Pseudomonas aeruginosa pose a serious threat to patients suffering from, among others, cystic fibrosis, chronic obstructive pulmonary disease, or bronchiectasis, often leading to life-threatening complications. The establishment of a chronic infection is substantially related to communication between bacteria via quorum-sensing networks. In this study, we aimed to assess the role of quorum-sensing signaling molecules of the Pseudomonas quinolone signal (POS) and to investigate the viscoelastic properties of lung tissue homogenates of PA-infected mice in a prolonged acute murine infection model. Therefore, a murine infection model was successfully established via intra-tracheal infection with alginate-supplemented Pseudomonas aeruginosa NH57388A. Rheological properties of lung homogenates were analyzed with multiple particle tracking (MPT) and quorumsensing molecules were quantified with LC-MS/MS. Statistical analysis of bacterial load and quorumsensing molecules showed a strong correlation between these biomarkers in infected lungs. This was accompanied by noticeable changes in the consistency of lung homogenates with increasing infection severity. Furthermore, viscoelastic properties of the lung homogenates strongly correlated with bacterial load and quorum sensing molecules. Considering the strong correlation between the viscoelasticity of lung homogenates and the aforementioned biomarkers, the viscoelastic properties of infected lungs might serve as reliable new biomarker for the evaluation of the severity of $P$. aeruginosa infections in murine models.

Carbapenem-resistant Pseudomonas aeruginosa is one of the most critical antibiotic-resistant bacteria as recently classified by the World Health Organization ${ }^{1}$, which is the reason why current anti-infective research is dedicated to the development of new treatment options against this pathogen ${ }^{2,3}$. Infections with $P$. aeruginosa lead to severe complications in chronic lung diseases like cystic fibrosis (CF), chronic obstructive pulmonary disease (COPD) and non-CF bronchiectasis (NCFB), often leading to significantly increased morbidity and mortality ${ }^{4,5}$. Especially

${ }^{1}$ Department of Drug Delivery (DDEL), Helmholtz-Institute for Pharmaceutical Research Saarland (HIPS)-Helmholtz Centre for Infection Research (HZI), Helmholtz Institute for Pharmaceutical Research Saarland, Campus E8.1, 66123 Saarbrücken, Germany. ${ }^{2}$ Department of Drug Design and Optimization (DDOP), Helmholtz-Institute for Pharmaceutical Research Saarland (HIPS)-Helmholtz Centre for Infection Research (HZI), Campus E8.1, 66123 Saarbrücken, Germany. ${ }^{3}$ German Centre for Infection Research (DZIF), Partner Site Hannover-Braunschweig, Saarbrücken, Germany. ${ }^{4}$ Department of Internal Medicine V - Pulmonology, Allergology, Critical Care Medicine, Saarland University Hospital, 66421 Homburg, Germany. ${ }^{5}$ Department of Pharmacy, Saarland University, Campus E8.1, 66123 Saarbrücken, Germany. ${ }^{6}$ Present address: Kusudama Therapeutics, Parque Científico y Tecnológico de Gipuzkoa, 20014 Donostia-San Sebastián, Spain. ${ }^{7}$ These authors contributed equally: Xabier Murgia and Andreas M. Kany. ${ }^{凶}$ email: martin.empting@helmholtz-hips.de; teresa.roehrig@ googlemail.com 
in CF, abnormal mucus morphology, characterized by increased thickness and volume, reduces mucociliary clearance, providing a favorable environment for bacterial colonization ${ }^{6}$. Despite antibiotic treatment, these infections can become persistent, causing recurring exacerbations and providing a high possibility for antibiotic resistance development ${ }^{4,5}$. In early stages of infection, $P$. aeruginosa is able to form impenetrable biofilms and in later stages of infection may further mutate to a mucoid phenotype, worsening the overall disease prognosis ${ }^{5}$. In order to establish and maintain host infections, $P$. aeruginosa upregulates the expression of several virulence factors including biofilm and additional alginate production via quorum-sensing (QS) systems. P. aeruginosa-specific transcriptional multi-virulence factor regulator ( $\mathrm{mvfR}$ ), also known as pqsR, is activated by the alkylquinolone PQS (2-heptyl-3-hydroxy-4(1H)-quinolone) and its biosynthetic precursor HHQ (2-heptyl-4-quinolone). As one of at least four QS systems in P. aeruginosa, pqsR mediates pyocyanin and hydrogen cyanide production as well as biofilm formation and alginate production in particular ${ }^{7,8}$. In CF diagnostics, the PQS precursor HHQ (2-heptyl$4(1 H)$-quinolone) can even be used to identify current $P$. aeruginosa infections from patients' plasma samples ${ }^{9}$.

In order to study P. aeruginosa infections in the context of chronic lung diseases, the use of animal models has become indispensable. Nevertheless, developing such chronically-infected, yet reproducible models with reliable readouts constitutes a great experimental challenge with just a few successful examples reported in the literature ${ }^{10,11}$. Facchini et al. utilized the non-mucoid clinical $P$. aeruginosa strain RP73 embedded in agar beads to chronically infect mice, while Hoffmann et al. used the mucoid strain NH57388A in alginate suspension also in mice. Common readouts for the infection status of these in vivo infection models embrace colony-forming unit (CFU) count, QS signal molecules, histopathological analysis, and immunological biomarkers. Additionally, Hoffmann et al. determined the production of endogenously produced bacterial alginate in lung homogenates. Bacterial alginate production and mucus hypersecretion are two well-known factors affecting the viscoelastic properties of the pulmonary lining fluid ${ }^{12}$.

Taking inspiration from the aforementioned models, our aim was to set-up an in vivo model of prolonged acute $P$. aeruginosa infection in order to study the role of pqsR and its signal molecules in lung infection. We opted for a sub-chronic model with a 72-h end point employing P. aeruginosa isolate NH57388A strain in alginate suspension as the inoculum applied via the intratracheal instillation (i.t.) route. In the early stages of model establishment, it became macroscopically apparent that lung homogenates generated from severely infected mice had a higher viscoelasticity compared to those corresponding to less severely infected animals. This observation prompted us to postulate that mechanical properties of lung homogenates in this model could correlate with the biomarker levels of infection severity. We therefore investigated the micro-rheological characteristics of lung homogenates by means of multiple particle tracking (MPT). This technique consists in tracking the Brownian motion of tracer particles dispersed within a complex biological fluid and allows determining the mechanical properties of the material by measuring the displacement of the tracer particles at different time scales ${ }^{13,14}$. To test our hypothesis, we investigated the correlation between CFU counts and the amount of pqsR molecules (PQS and HHQ) determined in the lung homogenates of mice infected with P. aeruginosa NH57388A with the mechanical properties of the same lung homogenates measured by means of MPT micro-rheology.

\section{Materials and methods}

Bacterial culture. Pseudomonas aeruginosa strain NH57388A was grown in lysogeny broth (LB) medium at $37^{\circ} \mathrm{C}$, overnight, $170 \mathrm{rpm}$ and adjusted to an $\mathrm{OD}_{600}=1$, and diluted $1: 1$ with sodium alginate solution (in $0.9 \%$ sterile $\mathrm{NaCl}$, final concentration $11 \mathrm{mg} / \mathrm{mL}$ ) to yield the desired bacterial density required for infection.

Mouse experiment. Mouse experiments were approved based on ethical considerations by the animal welfare committee of the 'Landesamt für Soziales, Gesundheit und Verbraucherschutz' of the State of Saarland in accordance with the national guidelines for animal treatment on August, 17th, 2017 (22/2017). In two different experiments, ten-week old female C57BL/6 N mice were slightly anesthetized intraperitoneally with $2.025 \mathrm{mg}$ of ketamine hydrochloride (Ursotamin, Serumwerk Bernburg, Germany) and $0.12 \mathrm{mg}$ of xylazine hydrochloride (Xylazin, WDF, Serumwerk Bernburg) per mouse. Lidocaine hydrochloride (Xylocain; Aspen Pharma, Irland) was used for local anesthesia of the epiglottis before endotracheal intubation with a flexible cannula and infection with $40 \mu \mathrm{L}$ viable $P$. aeruginosa NH57388A culture. Bacterial load was determined to be $5 \times 10^{7} \mathrm{CFU}$ by plating serial dilutions on LB-agar plates. During the course of $72 \mathrm{~h}$ after infection, a high weight loss was observed (mean $20 \pm 1.5 \%$ ) at a survival rate of $83 \%$, whilst the overall appearance of the animals stayed within the predefined scores for animal distress. $24 \mathrm{~h}(\mathrm{~N}=1), 48 \mathrm{~h}(\mathrm{~N}=1)$, and $72 \mathrm{~h}(\mathrm{~N}=10)$ after infection, mice were euthanized, the lungs removed and immediately stored on ice. They were then homogenized with an ultra turrax homogenizer (IKA, Staufen im Breisgau, Germany) at 20,000 rpm in $500 \mu \mathrm{L}$ ice-cold sterile phosphate buffered saline and immediately stored at $-80^{\circ} \mathrm{C}$ until analysis.

LC-MS/MS analysis of alkylquinolones. Alkylquinolone signal molecules PQS (2-heptyl-3-hydroxy4-quinolone), HHQ (2-heptyl-4-quinolone), HQNO (N-oxo-2-heptyl-4-Hydroxyquinoline) in murine lung homogenates were analyzed via LC-MS/MS. Stable isotope standards (d4HHQ and d4PQS, 200 pmol each) were added to lung homogenates prior to extraction with $1 \mathrm{~mL}$ ethyl acetate. After extraction under vigorous shaking in an orbital shaker (10 min, $1750 \mathrm{rpm}$, IKA, Staufen, Germany) $800 \mu \mathrm{L}$ supernatant was dried and reconstituted with $400 \mu \mathrm{L}$ methanol. Lower limits of quantification were determined to be $0.25 \mathrm{pmol} / \mathrm{lung}$ for HHQ and HQNO, $2.5 \mathrm{pmol} / \mathrm{lung}$ for PQS. LC-ESI-MS/MS conditions were as follows: Dionex Ultimate 3000 HPLC coupled to triple quad mass spectrometer TSQ Quantum Access Max (Thermo Fisher Scientific, Waltham, MA, USA), Zorbax Eclipse XDB $80 \AA$ C18 $5 \mu \mathrm{m} \mathrm{4.6 \times 50} \mathrm{mm} \mathrm{equipped} \mathrm{with} \mathrm{guard} \mathrm{column} \mathrm{(Agilent,} \mathrm{Santa} \mathrm{Clara,}$ CA, USA); eluent $\mathrm{A}-\mathrm{H}_{2} \mathrm{O}$ with $0.1 \%$ trifluoroacetic acid (TFA), $0.1 \%$ pentafluoropropionic acid (PFPA), $0.1 \%$ heptafluorobutyric acid (HFBA); eluent B-acetonitrile with $0.1 \%$ TFA, $0.1 \%$ PFPA, $0.1 \%$ HFBA; isocratic 50\% 
a

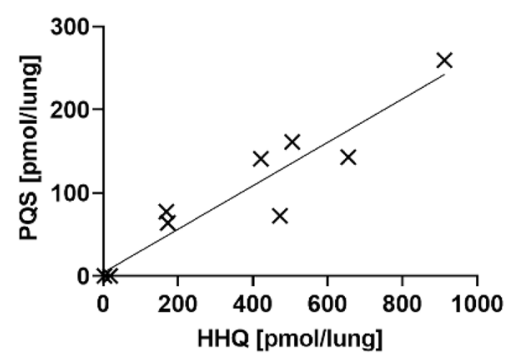

b

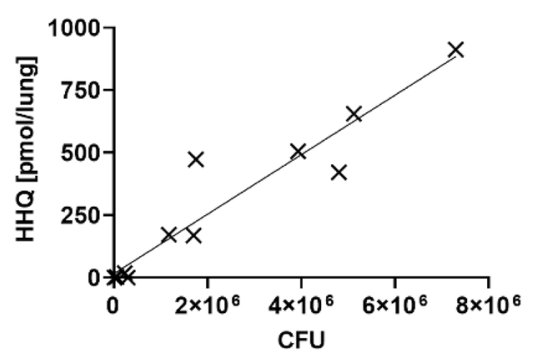

C

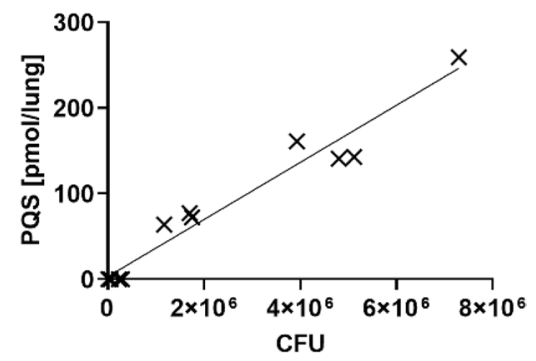

Figure 1. Dependence of signal molecule production on bacterial load. (a) Scatterplot and linear regression of the quorum sensing signaling molecules PQS (2-heptyl-3-hydroxy-4(1H)-quinolone) vs HHQ (2-heptyl-4quinolone) detected in murine Pseudomonas aeruginosa NH57388A lung infection samples. (b,c) Scatterplot and linear regression of PQS (B) and HHQ (C) vs corresponding colony forming units (CFU). See also Fig. S1.

A; flow- $0.7 \mathrm{~mL} / \mathrm{min}$. Instrument parameters: Spray voltage $3500 \mathrm{~V}$, vaporizer temperature $370{ }^{\circ} \mathrm{C}$, sheath gas pressure $35 \mathrm{psi}$, aux gas pressure $30 \mathrm{psi}$, capillary temperature $270^{\circ} \mathrm{C}$, collision pressure $1.5 \mathrm{mTorr}$, positive ionization mode. For QS analyte reaction monitoring parameters please see Table S1.

Multiple particle tracking. Red-fluorescent, carboxyl-coated polystyrene tracer particles with a nominal size of $200 \mathrm{~nm}$ were purchased from Invitrogen. The surface of the particles was modified by coating them with a dense layer of polyethylene glycol (PEG, $5000 \mathrm{Da})$, as previously described ${ }^{15}$. Briefly, a $0.2 \%(w / w)$ particle suspension was diluted eightfold in ultrapure water. An excess of 4-(4,6-dimethoxy-1,3,5-triazin-2-yl)-4-methylmorpholinium chloride (DMTMM) was added to the particle suspension. Following $3 \mathrm{~h}$-activating reaction, an excess amount of methoxy-PEG-amine, prepared in ultrapure water at $4 \%(w / w)$, was added. The resulting solution was carried out at room temperature for $24 \mathrm{~h}$. The particle suspension was then centrifuged and washed 3 times with ultrapure water. The particles were re-suspended in ultrapure water to a $0.2 \%(w / w)$ concentration. PEG-coated particle suspensions were characterized in terms of particle diameter and surface charge by nanoparticle tracking analysis (NTA, Nanosight, Malvern Instruments) and dynamic light scattering (Z-sizer Nano ZSP, Malvern Instruments), respectively. Mean hydrodynamic particle size slightly increased after PEGylation from $192 \pm 28 \mathrm{~nm}$ to $218 \pm 48 \mathrm{~nm}$ (mean \pm standard deviation). The surface charge was determined in $1 \mathrm{mM}$ $\mathrm{NaCl}$ solution and showed an increase after PEGylation from $-24 \pm 0.83 \mathrm{mV}$ to neutral charge $(-0.2 \pm 1.45 \mathrm{mV})$ indicative of successful surface PEG coating of the particles.

Lung homogenates were thawed gradually and allowed to reach room temperature. $30 \mu \mathrm{L}$ of each lung homogenate sample were mixed with $1 \mu \mathrm{L}$ of the tracer particle stock $(0.1 \% \mathrm{w} / \mathrm{v})$. The whole mixture was transferred to a chamber mounted onto a microscope slide (Gene Frame, Thermo Fisher Scientific) and sealed with a cover slip. Samples were incubated for at least one hour before imaging. Video sequences were captured with a confocal laser scanning microscope (Leica TCS SP 8; Leica, Mannheim, Germany) equipped with a DPSS 561 laser and a HC PL APO CS $63 \times$ objective (NA of 1.2). Experiments were performed at $23^{\circ} \mathrm{C}$. For each sample two independent fields were recorded at a resolution of $0.142 \mu \mathrm{m}$ per pixel and a frame rate of 28 frames per second. The physical length of each microscopic field represents an area of $147 \times 37 \mu \mathrm{m}$. Image processing and analysis were performed as described by Ho et al. ${ }^{15}$.

Statistical analysis. Regression curves shown in Figs. 1, 3, Figs. S1, S4 and the corresponding Pearson's correlation coefficients ( $r$, Table S2 and S3) were determined using the simple linear regression function of GraphPad Prism 8.4.2 (GraphPad Software, San Diego, CA, USA). Histograms in Figs. 2, Figs. S2 and S3 were created using the frequency distribution function of Prism. The presented coefficients were determined from 12 individual samples, which can be separated into two independent experiments with 4 and 8 individual samples, respectively. Separate values for $r$ representing correlation in the individual experiment as shown in Figs. S1 and S4 were determined using the same procedure. Individual histograms are presented in Figs. S2 and S3.

\section{Results and discussion}

Bacterial load of mouse lungs strongly correlates with signal-molecule levels. In order to study chronic $P$. aeruginosa infections of the lung and the role of the pqsR quorum sensing system, a chronic murine lung infection model was established in accordance with Hoffmann et al. ${ }^{11}$. In the present study, infection in mice was accomplished via an alginate-based intra-tracheal inoculation with mucoid P. aeruginosa NH57388A. Upon removal of the murine lungs $72 \mathrm{~h}$ post infection, heterogeneously distributed lesions of the lung tissue were macroscopically visible. Throughout homogenization and sample-extraction procedures we noticed different consistencies of lung homogenates for individual animals. In significant cases, lung homogenates were not of a fluid consistency and showed an apparent higher viscosity and an elastic behavior compared to other samples.

The median CFU count in extracted organs was $1.4 \times 10^{6} \mathrm{CFU} /$ lung, showing a wide range across the animals (range $\left.=7.4 \times 10^{3}-7.3 \times 10^{6}\right)$, which indicates a high variance of infection status in individual mice. In regard to the inoculum, the CFU count shows a high clearance of bacteria during $72 \mathrm{~h}$ post infection while a severe infection status is maintained in most cases based on weight loss and animal behavior. The individual variability 
a

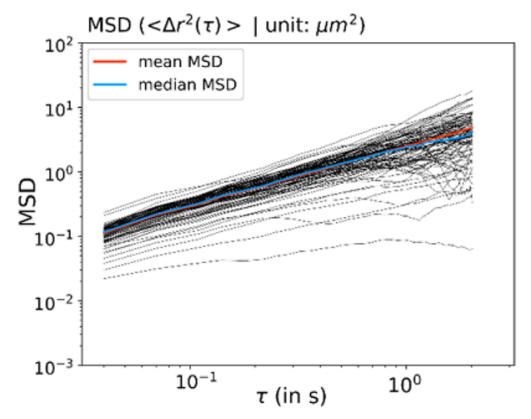

d

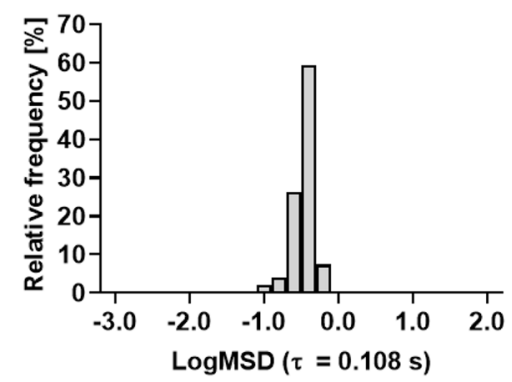

g

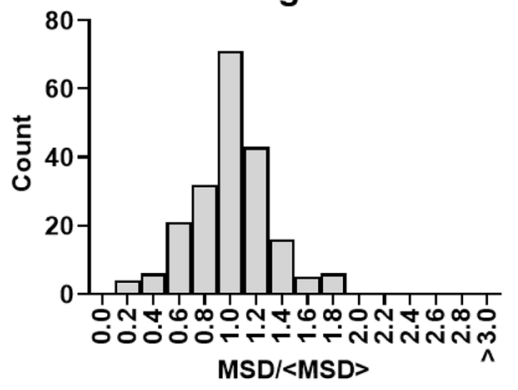

b

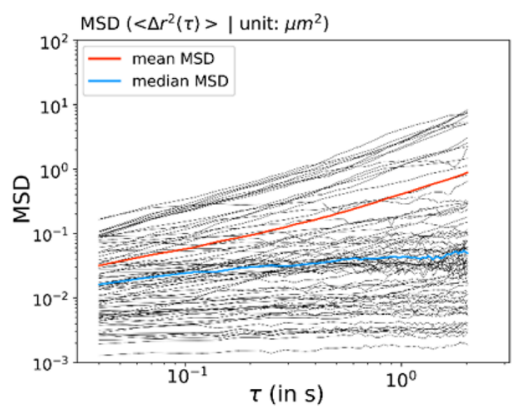

e

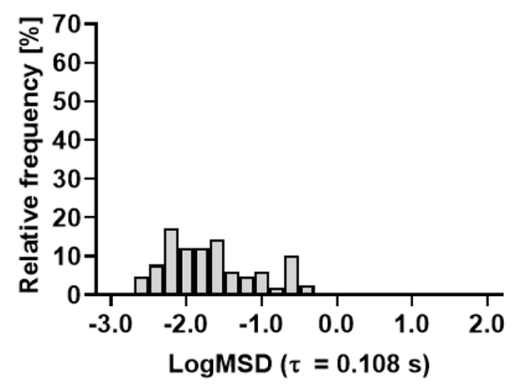

h

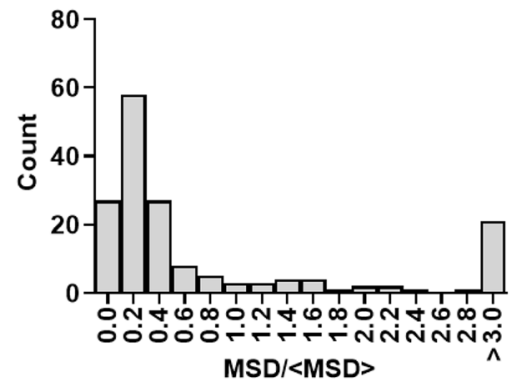

C
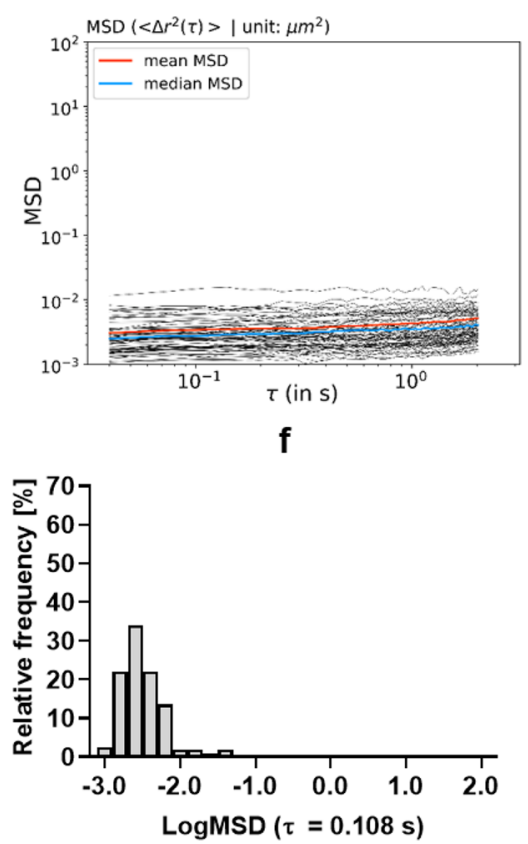

i

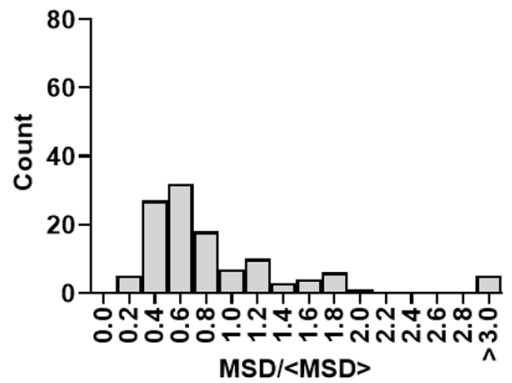

Figure 2. Representative mean squared displacement (MSD) plots as a function of the time scale $(\tau)$ of polyethylene glycol-coated polystyrene particles. (a-c) mean squared displacement (MSD) of each individual tracer particle trajectory at different $\tau$ in representative lung homogenates of murine Pseudomonas aeruginosa NH57388A lung infection samples. All animals were infected with the same bacterial inoculum but developed different degrees of infection that correlated with low (a), medium (b), and high (c) viscoelasticity. The mean and the median of all individual particles are indicated by the red and blue lines, respectively. (d-f) corresponding distribution of the $\log$ MSD at a $\tau=0.1 \mathrm{~s}$. See also Fig. S2. Tracer particles had a mean diameter of $218 \mathrm{~nm}$. (g-i) Representative mean squared displacement (MSD) distributions at $\tau=0.108 \mathrm{~s}$ normalized by the ensemble-average MSD (〈MSD) at $\tau=0.108$ s. See also Fig. S3.

observed in terms of CFU count was mirrored by the QS signal molecules quantified in lung homogenates. HHQ and PQS correlated strongly $(\mathrm{r}=0.9548$, Fig. 1a), as was expected. Since QS is active in high cell densities, we anticipated further that more signal molecules could be found in those lung homogenates with the highest CFU counts. Indeed, Fig. 1b,c shows a positive correlation of CFU counts with HHQ $(r=0.9523)$ and PQS $(r=0.9769)$, confirming this hypothesis and validating the infection model. To the best of our knowledge, this is the first time that a very strong correlation between PQS or HHQ and bacterial load in biological samples has been demonstrated. In two studies by Barr and coworkers ${ }^{9,16}$, the correlation of HHQ concentration in plasma samples from CF patients with bacterial load of sputum was significant in a moderate to weak range $(r=0.28$ and $r=0.51)$ and in a study by Collier and coworkers, a correlation between PQS and bacterial density in sputum samples from CF patients was shown but not quantitatively ${ }^{17}$. Considering the mucoid feature of the P. aeruginosa NH57388A strain, we investigated whether the determined bacterial load and the detected signal molecule levels could further be correlated with the mechanical properties of the lung homogenate.

Micro-rheology of tissue homogenates serves as reliable biomarker. A volume of $30 \mu \mathrm{L}$-aliquots of individual lung homogenates were mixed with red-fluorescent, $200 \mathrm{~nm}$ particles coated with a dense layer of polyethylene glycol (PEGylation), and their Brownian motion was monitored by fluorescence video microscopy. Particle PEGylation is a well-established nanoparticle coating strategy that reduces the interaction between particles and biological entities ${ }^{18,19}$. Therefore, it is assumed that such particles are bio-inert and that those particles, 
which appear immobile in video microscopy are sterically hindered rather than chemically adsorbed. The mean squared displacement (MSD or $\left\langle\Delta r^{2}\right.$ ) of the tracer particles at each time scale $(\tau)$ was determined from video microscopy images by computing the $\mathrm{x}-\mathrm{y}$ coordinates of each particle in each video frame using a customwritten Python script, as previously described ${ }^{15,20}$ :

$$
\left\langle\Delta r^{2}(\tau)\right\rangle=\{x(t+\tau)-x(t)\}^{2}+\{y(t+\tau)-y(t)\}^{2}
$$

Figure 2a-c depicts MSD plots of representative lung tissue homogenates with different macroscopic consistencies (low, medium and high apparent viscoelasticty, respectively). Figure $2 \mathrm{~d}-\mathrm{f}$ describes the $\log \mathrm{MSD}$ distribution of these samples at a $\tau$ of $0.108 \mathrm{~s}$. Particles with a diffusive behavior increase their MSD as a function of the $\tau$ (Fig. 2a, video S1), whereas those particles trapped by the biomacromolecular matrix of the lung homogenate show a constant MSD at any $\tau$ (Fig. 2c, video S2). Lung homogenates simultaneously displaying viscous and elastic areas show MSDs corresponding to diffusive and hindered particle trajectories (Fig. 2b, video S3). From the log-log MSD plots, the slope $(\alpha)$ of each particle trajectory can be calculated:

$$
\alpha=\frac{\mathrm{d} \log \left\langle\Delta r^{2}\right\rangle(\tau)}{\mathrm{d} \log (\tau)}
$$

The slope describes the diffusive properties of the tracer particles. For particles diffusing through a Newtonian fluid $\alpha=1$. Conversely, for particles immobilized by an elastic environment $\alpha=0^{21}$.

The wide variability observed for the CFU counts and QS signal molecules was also evidenced by the microrheology study of the lung homogenates. The displacement of the tracer particles as well as the slope of the MSD trajectories varied significantly across the 12 independent lung samples analyzed in this study. The range of the mean $\log \mathrm{MSD}(\tau=0.108 \mathrm{~s}$ ) expanded from -0.44 to -2.49 corresponding to samples with the most diffusive and immobile particles, respectively (Fig. S2). Similarly, the mean $\alpha$ at a $\tau=0.108$ s ranged between 0.94 , for the sample with the most diffusive particles, and 0.11 for the most elastic sample. This wide range of MSD and $\alpha$ across individual samples implies different cross-linking densities between the constituents of the lung homogenates of different animals. Such differences clearly point to a different biochemical composition of the independent lung homogenates. In those lung homogenates corresponding to the most severe infection status (i.e. higher CFU), tracer particles appear almost exclusively immobilized, which indicates the existence of an interconnected biomacromolecular network with a pore size below the diameter of the tracer particles. Additionally, micro-scale inhomogeneity was detected in individual samples, which simultaneously showed diffusive and immobile particles. To quantify the level of inhomogeneity, the MSD distributions at $\tau=0.108 \mathrm{~s}$ normalized by the ensemble-average MSD (〈MSD at $\tau=0.108 \mathrm{~s}$ ) were plotted ${ }^{13}$. Figure $2 \mathrm{~g}-\mathrm{i}$ shows three representative MSD/ 〈MSD distribution plots corresponding to the same examples as in Fig. 2a-f:

For the sample with an apparent low viscoelasticity, the distribution is symmetric about the mean, corresponding to a homogeneous fluid. Conversely, for the samples with apparent intermediate and high viscoelasticity no symmetry can be observed and most trajectories appear to the left side of the mean, indicative of sample inhomogeneities at the micro-scale where diffusive, sub-diffusive and immobile particle trajectories can be observed.

The diffusion coefficient (D) was calculated for particle trajectories with an $\alpha>0.8$ :

$$
\left\langle\Delta r^{2}(\tau)\right\rangle=4 \mathrm{D} \tau
$$

which was further used to calculate the viscosity $(\eta)$ of the material surrounding the tracer particles by applying the Stokes-Einstein relation:

$$
\eta=\frac{k_{B} T}{3 d \pi D}
$$

where $d$ is the particle diameter, $K_{B}$ the Boltzmann constant and $T$ the temperature.

The thermal motion of non-interacting immobile particles is determined by the elastic properties of the surrounding network. Therefore, for particle trajectories with an $\alpha<0.2$ the so-called plateau modulus $\mathrm{G}_{0}$ of this network was estimated using the following expression ${ }^{22}$ :

$$
G_{0}=\frac{2 K_{B} T}{3 \mathrm{r} \pi\left\langle\Delta r^{2}\right\rangle}
$$

where $\left\langle\left\langle\Delta r^{2}\right\rangle\right.$ is the time independent average MSD and $r$ is the radius of the tracer particles.

According to the classical theory of rubber elasticity, the modulus $\mathrm{G}_{0}$ can be related to the mesh size $\xi$ of the elastic network characterizing the average distance between adjacent crosslinks in the network ${ }^{23}$.

$$
\xi=\left(K_{B} T / G_{0}\right)^{1 / 3}
$$

The results from the MPT analysis are summarized in Table 1.

In three lung homogenates out of twelve infected mice samples, there were not enough diffusive particles $(\alpha>0.8)$ to compute $\mathrm{D}$ or $\eta$. These samples displayed the lowest mean $\alpha$ values $(0.11-0.20)$, the highest $\mathrm{G}_{0}$ $(2.48-3.03 \mathrm{~Pa})$, and a pore size below $130 \mathrm{~nm}$. The $\eta$ of the lung homogenate fluid surrounding the tracer particles could be determined from nine out of twelve infected mice samples and it ranged between 2.41 and $6.22 \mathrm{mPa}^{*} \mathrm{~s}$, representing two- and sixfold the viscosity of water at $20^{\circ} \mathrm{C}\left(1 \mathrm{mPa}^{*} \mathrm{~s}\right)$.

Remarkably, the mean logMSD and $\alpha$ determined by particle tracking micro-rheology showed a strong linear correlation with CFU, PQS and HHQ (Fig. 3) with the absolute values of the Pearson's correlation coefficients 


\begin{tabular}{|c|c|c|c|c|c|c|}
\hline & \begin{tabular}{|l|l|} 
MSD \\
$\boldsymbol{\tau}=\mathbf{0 . 1 0 8} \mathrm{s}$
\end{tabular} & $\begin{array}{l}\mathrm{D} \\
\tau=0.108 \mathrm{~s}\end{array}$ & $\alpha$ & $\eta$ & $G_{0}$ & $\xi$ \\
\hline & {$\left[\mu \mathbf{m}^{2}\right]$} & {$\left[\mu \mathrm{m}^{2} / \mathrm{s}\right]$} & & {$\left[\mathrm{mPa}^{*} \mathrm{~s}\right]$} & {$[\mathrm{Pa}]$} & {$[\mathrm{nm}]$} \\
\hline Mouse \#1 & $0.368 \pm 0.12$ & $0.901 \pm 0.26$ & $0.94 \pm 0.11$ & $2.41 \pm 0.75$ & a & a \\
\hline Mouse \#2 & $0.376 \pm 0.10$ & $0.891 \pm 0.22$ & $0.93 \pm 0.11$ & $2.41 \pm 0.88$ & a & a \\
\hline Mouse \#3 & $0.348 \pm 0.10$ & $0.828 \pm 0.21$ & $0.91+0.12$ & $2.60 \pm 0.85$ & a & $\mathrm{a}$ \\
\hline Mouse \#4 & $0.228 \pm 0.16$ & $0.764 \pm 0.23$ & $0.73 \pm 0.34$ & $3.03 \pm 1.99$ & $1.57 \pm 1.07$ & $157 \pm 52$ \\
\hline Mouse \#5 & $0.161 \pm 0.17$ & $0.794 \pm 0.29$ & $0.61 \pm 0.31$ & $3.05 \pm 1.9$ & $0.75 \pm 0.59$ & $208 \pm 72$ \\
\hline Mouse \#6 & $0.277 \pm 0.16$ & $0.746 \pm 0.35$ & $0.79 \pm 0.22$ & $3.32 \pm 1.58$ & a & a \\
\hline Mouse \#7 & $0.182 \pm 0.16$ & $0.673 \pm 0.33$ & $0.72 \pm 0.32$ & $4.23 \pm 3.60$ & $1.56 \pm 0.90$ & $146 \pm 24$ \\
\hline Mouse \#8 & $0.057 \pm 0.09$ & $0.520 \pm 0.22$ & $0.34 \pm 0.31$ & $5.03 \pm 3.07$ & $1.16 \pm 1.01$ & $179 \pm 59$ \\
\hline Mouse \#9 & $0.086 \pm 0.11$ & $0.512 \pm 0.24$ & $0.47 \pm 0.35$ & $6.22 \pm 6.03$ & $1.75 \pm 1.40$ & $160 \pm 54$ \\
\hline Mouse \#10 & $0.004 \pm 0.005$ & $\mathrm{~b}$ & $0.11 \pm 0.10$ & $\mathrm{~b}$ & $3.03 \pm 1.51$ & $118 \pm 24$ \\
\hline Mouse \#11 & $0.006 \pm 0.005$ & b & $0.20 \pm 0.17$ & b & $2.84 \pm 1.67$ & $123 \pm 27$ \\
\hline Mouse \#12 & $0.006 \pm 0.010$ & b & $0.13 \pm 0.12$ & b & $2.48 \pm 1.21$ & $129 \pm 39$ \\
\hline
\end{tabular}

Table 1. Summary of multiple particle tracking (MPT) analysis of each lung homogenate from 12 independent mice. $\langle\mathrm{MSD}$, ensemble-averaged mean squared displacement from all trajectories; $D$ diffusion; $\alpha$ exponential coefficient; $\eta$ viscosity; $G_{o}$ plateau modulus; $\xi$ mess size. D and $\eta$ were average from all trajectories with $\alpha>0.8$, whereas $G_{o}$ and $\xi$ were averaged from trajectories with $\alpha<0.2$. ${ }^{a}$ Not enough trajectories (less than

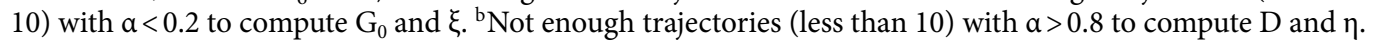
Mean \pm Standard deviation are shown.

a
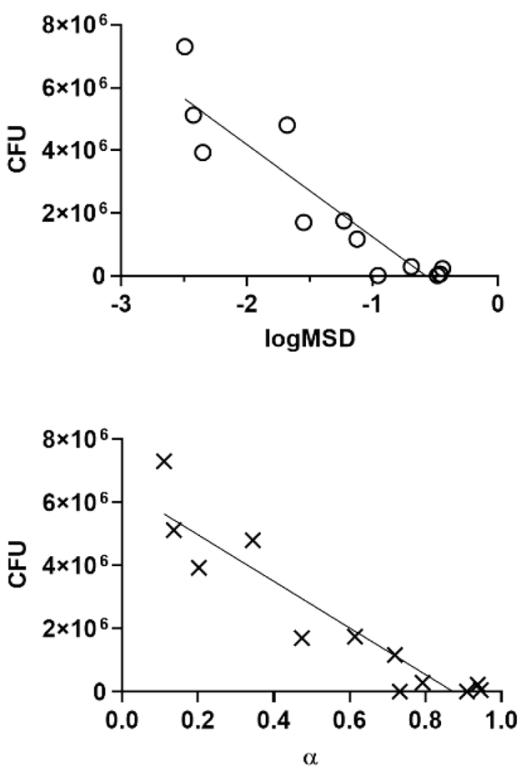

b
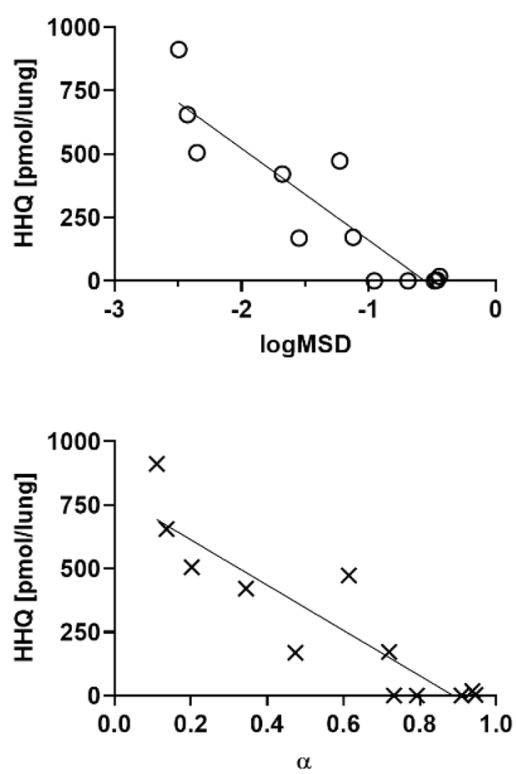

C
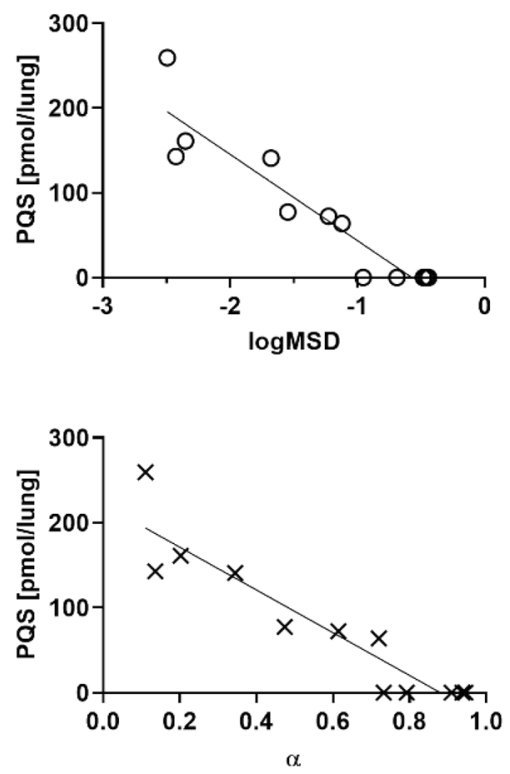

Figure 3. Rheological properties strongly correlate with bacterial biomarkers. Scatterplot and linear regression of colony forming units (CFU; a), PQS (b) and HHQ (c) $v$ s tracer particle logMSD (logarithm of mean square displacement at $\tau=0.1 \mathrm{~s}$ ) and exponential coefficient $\alpha$. See also Fig. S4.

being greater than 0.90 in all cases. Consequently, the grade of infection of this particular model can be accurately estimated by determining the micro-rheological properties of lung homogenates. The MPT analysis revealed the different response to infection of each mouse. Even though all animals received the same inoculum of $P$. aeruginosa, the lung homogenates of mice with the lowest bacterial load (i.e. lowest CFU) displayed mean $\alpha$ values higher than 0.9 and the tracer particles appeared to be diffusing through a viscous fluid. Conversely, in those mice with the highest bacterial load (i.e. highest CFU), the tracer particles were almost exclusively immobilized by a biomolecular network with a pore size of approximately $200 \mathrm{~nm}$. The strict pore size might have implications for nanoparticle-mediated drug delivery and may also limit the migration of immune cells in the setting of severe pneumonia.

The samples analyzed in this experiment represent tissue homogenates obtained from two independent experimental sessions with 4 or 8 mice in each session, respectively. Within each replicate, there is a similarly 
strong correlation between signaling molecules, CFU and $\log \mathrm{MSD} / \alpha(\mathrm{r}>0.87$, Fig. S4 and a Table S3), showing reproducibility of our results.

These findings demonstrate our hypothesis that the mechanical properties of lungs from infected mice serve as reliable marker to assess the status of an infection with $P$. aeruginosa. Several factors may account for the overall increase of the viscosity in the setting of bacterial infections aside from the alginate overproduced by mucoid $P$. aeruginosa strains. For instance, high bacterial content, mucus hypersecretion, increased mucin cross-linking, and the development of a parallel network of DNA debris and cytoskeleton proteins from apoptotic neutrophils significantly increase the viscosity of CF sputum ${ }^{24-27}$. Our MPT investigations and those of others have shown that a significant fraction of $200 \mathrm{~nm}$ PEGylated particles (35-90\%) display a diffusive behavior through pulmonary mucus and CF sputum ${ }^{15,19,28}$. It is noteworthy that in 3 out of 12 samples of this work almost $100 \%$ of the PEGylated tracer particles were immobilized by a biomacromolecular network. In vitro, $P$. aeruginosa biofilms show rheologically inhomogeneous structures at the microscale derived from the adhesion to the growth substrate, the arrangement of individual bacteria, and the formation of larger, multibacterial floc-like structures ${ }^{29}$. Moreover Gloag et al. have recently shown that $P$. aeruginosa mucoid biofilms progress to an elastic-solid behavior over time, proposing the viscoelasticity as a virulence factor of biofilms ${ }^{30}$. However, here we did not exclusively analyze the pulmonary lining fluid or the bacterial biofilms alone but the whole lung content, including lung tissue, after homogenization. Nevertheless, considering that particles dispersed in lung homogenates from animals with a mild infection showed a diffusive behavior, we hypothesize that the changes in the micro-rheological properties of the lung homogenates are principally associated with the proliferation of mucoid biofilms of the $P$. aeruginosa NH57388A alginate-hypersecreting strain.

\section{Conclusion}

This study initially aimed to establish a murine sub-chronic lung infection model with mucoid $P$. aeruginosa NH57388A. Not only chronic but also acute murine lung infection models are difficult to establish and maintain due to high variances of survival and biomarkers within the infected population as well as between experiments. In the present study an alginate based murine lung infection model in a prolonged acute setting was successfully used. Although the biomarkers HHQ, PQS and CFU showed high variance, their positive correlation was very strong as was hypothesized. To the best of our knowledge, only weak to moderate positive correlations have been demonstrated before. Additionally, in the present study micro-rheological properties of lung homogenates were analyzed and shown to strongly correlate with HHQ (or PQS) and CFU as well. Especially in the case of NCFB, clotting of the patient's bronchi by infection-mediated mucus plugs is a major parameter affecting organ function and causing pulmonary exacerbations. Although it is not known whether the aforementioned correlation stems from alginate overproduction of the mucoid strain or mucus overproduction of the host, gaining access to this clinically relevant study endpoint is of high relevance for (pre-) clinical development of mucolytic and nontraditional anti-infective drugs (e.g. pathoblockers). The data provided herein shed light on the potential of lung homogenate viscosity determined via MPT as an alternative study endpoint for assessing the effectiveness of nonantibiotic therapies against $P$. aeruginosa infections in in vivo models with only small sample volumes required.

Received: 5 February 2020; Accepted: 27 August 2020

Published online: 05 October 2020

\section{References}

1. World Health Organization. Antibacterial Agents in Clinical Development. An Analysis of the Antibacterial Clinical Development Pipeline (Including Tuberculosis, World Health Organization, Geneva, 2017).

2. Schütz, C. \& Empting, M. Targeting the Pseudomonas quinolone signal quorum sensing system for the discovery of novel antiinfective pathoblockers. Beilstein J. Org. Chem. 14, 2627-2645 (2018).

3. Kamal, A. A. M. et al. Quorum sensing inhibitors as pathoblockers for Pseudomonas aeruginosa infections: a new concept in antiinfective drug discovery. In Antibacterials (eds Fisher, J. F. et al.) 185-210 (Springer International Publishing, Berlin, 2018).

4. Finch, S., McDonnell, M. J., Abo-Leyah, H., Aliberti, S. \& Chalmers, J. D. A comprehensive analysis of the impact of Pseudomonas aeruginosa colonization on prognosis in adult bronchiectasis. Ann. Am. Thorac. Soc. 12, 1602-1611 (2015).

5. Silva Filho, L. V. R. et al. Pseudomonas aeruginosa infection in patients with cystic fibrosis: scientific evidence regarding clinical impact, diagnosis, and treatment. J. Bras. Pneumol. 39, 495-512 (2013).

6. Williams, O. W., Sharafkhaneh, A., Kim, V., Dickey, B. F. \& Evans, C. M. Airway mucus: from production to secretion. Am. J. Respir. Cell Mol. Biol. 34, 527-536 (2006).

7. Dubern, J.-F. \& Diggle, S. P. Quorum sensing by 2-alkyl-4-quinolones in Pseudomonas aeruginosa and other bacterial species. Mol. Biosyst. 4, 882-888 (2008).

8. Lee, J. \& Zhang, L. The hierarchy quorum sensing network in Pseudomonas aeruginosa. Protein Cell 6, 26-41 (2015).

9. Barr, H. L. et al. Diagnostic and prognostic significance of systemic alkyl quinolones for P. aeruginosa in cystic fibrosis: a longitudinal study. J. Cyst. Fibros. 16, 230-238 (2017).

10. Facchini, M., de Fino, I., Riva, C. \& Bragonzi, A. Long term chronic Pseudomonas aeruginosa airway infection in mice. J. Vis. Exp. 85, e5109 (2014).

11. Hoffmann, N. et al. Novel mouse model of chronic Pseudomonas aeruginosa lung infection mimicking cystic fibrosis. Infect. Immun. 73, 2504-2514 (2005).

12. Lai, S. K., Wang, Y.-Y., Wirtz, D. \& Hanes, J. Micro- and macrorheology of mucus. Adv. Drug Deliv. Rev. 61, 86-100 (2009).

13. Oelschlaeger, C., Willenbacher, N. \& Neser, S. Multiple-particle tracking (MPT) measurements of heterogeneities in acrylic thickener solutions. In Surface and Interfacial Forces: From Fundamentals to Applications Progress in Colloid and Polymer Science (eds Auernhammer, G. K. et al.) 74-79 (Springer, Berlin, 2008).

14. Schuster, B. S., Suk, J. S., Woodworth, G. F. \& Hanes, J. Nanoparticle diffusion in respiratory mucus from humans without lung disease. Biomaterials 34, 3439-3446 (2013).

15. Ho, D.-K. et al. Farnesylated glycol chitosan as a platform for drug delivery: synthesis, characterization, and investigation of mucus-particle interactions. Biomacromol 19, 3489-3501 (2018). 
16. Barr, H. L. et al. Pseudomonas aeruginosa quorum sensing molecules correlate with clinical status in cystic fibrosis. Eur. Respir. J. 46, 1046-1054 (2015).

17. Collier, D. N. et al. A bacterial cell to cell signal in the lungs of cystic fibrosis patients. FEMS Microbiol. Lett. 215, 41-46 (2002).

18. Abuchowski, A., McCoy, J. R., Palczuk, N. C., van Es, T. \& Davis, F. F. Effect of covalent attachment of polyethylene glycol on immunogenicity and circulating life of bovine liver catalase. J. Biol. Chem. 252, 3582-3586 (1977).

19. Suk, J. S., Xu, Q., Kim, N., Hanes, J. \& Ensign, L. M. PEGylation as a strategy for improving nanoparticle-based drug and gene delivery. Adv. Drug Deliv. Rev. 99, 28-51 (2016).

20. Huck, B. C. et al. Macro- and microrheological properties of mucus surrogates in comparison to native intestinal and pulmonary mucus. Biomacromol 20, 3504-3512 (2019).

21. Murgia, X. et al. Size-limited penetration of nanoparticles into porcine respiratory mucus after aerosol deposition. Biomacromol 17, 1536-1542 (2016).

22. Wirtz, D. Particle-tracking microrheology of living cells: principles and applications. Annu. Rev. Biophys. 38, 301-326 (2009).

23. Kowalczyk, A., Oelschlaeger, C. \& Willenbacher, N. Visualization of micro-scale inhomogeneities in acrylic thickener solutions: a multiple particle tracking study. Polymer 58, 170-179 (2015).

24. Elborn, J. S. Cystic fibrosis. Lancet 388, 2519-2531 (2016).

25. Perks, B. \& Shute, J. K. DNA and actin bind and inhibit interleukin-8 function in cystic fibrosis sputa: in vitro effects of mucolytics. Am. J. Respir. Crit. Care Med. 162, 1767-1772 (2000).

26. Yuan, S. et al. Oxidation increases mucin polymer cross-links to stiffen airway mucus gels. Sci. Transl. Med. 7, 276ra27 (2015).

27. Murgia, X., Loretz, B., Hartwig, O., Hittinger, M. \& Lehr, C.-M. The role of mucus on drug transport and its potential to affect therapeutic outcomes. Adv. Drug Deliv. Rev. 124, 82-97 (2018).

28. Schuster, B. S., Ensign, L. M., Allan, D. B., Suk, J. S. \& Hanes, J. Particle tracking in drug and gene delivery research: state-of-the-art applications and methods. Adv. Drug Deliv. Rev. 91, 70-91 (2015).

29. Rogers, S. S., van der Walle, C. \& Waigh, T. A. Microrheology of bacterial biofilms in vitro: Staphylococcus aureus and Pseudomonas aeruginosa. Langmuir 24, 13549-13555 (2008).

30. Gloag, E. S., German, G. K., Stoodley, P. \& Wozniak, D. J. Viscoelastic properties of Pseudomonas aeruginosa variant biofilms. Sci. Rep. 8, 9691 (2018).

\section{Acknowledgements}

This work was financially supported by the Helmholtz Association through Helmholtz Validation Fund (HVF0054) and the German Centre for Infection Research (DZIF) through DZIF Flexible Funds (09.908).

\section{Author contributions}

T.R. and M.E. designed the study and planned the experiments. T.R. and C.H. established and performed the animal model. T.R. performed the LC-MS-based biomarker analysis. A.M.K. performed the sample preparation for MPT and the statistical analysis. X.M. performed and analyzed all MPT measurements. D.K.H. prepared the nanoparticles used in this study. C.DR. set-up the confocal microscopy technique. R.B., C.M.L., A.K.H.H. and R.W.H. supervised the project and supplied funding. X.M., A.M.K. and T.R. wrote the manuscript with contributions from all authors. All authors approved the final version of the manuscript.

\section{Funding}

Open Access funding enabled and organized by Projekt DEAL.

\section{Competing interests}

The authors declare no competing interests.

\section{Additional information}

Supplementary information is available for this paper at https://doi.org/10.1038/s41598-020-73459-5.

Correspondence and requests for materials should be addressed to M.E. or T.R.

Reprints and permissions information is available at www.nature.com/reprints.

Publisher's note Springer Nature remains neutral with regard to jurisdictional claims in published maps and institutional affiliations.

(c) (i) Open Access This article is licensed under a Creative Commons Attribution 4.0 International License, which permits use, sharing, adaptation, distribution and reproduction in any medium or format, as long as you give appropriate credit to the original author(s) and the source, provide a link to the Creative Commons licence, and indicate if changes were made. The images or other third party material in this article are included in the article's Creative Commons licence, unless indicated otherwise in a credit line to the material. If material is not included in the article's Creative Commons licence and your intended use is not permitted by statutory regulation or exceeds the permitted use, you will need to obtain permission directly from the copyright holder. To view a copy of this licence, visit http://creativecommons.org/licenses/by/4.0/.

(C) The Author(s) 2020 